\section{Brustkrebs: Wann adjuvantes Docetaxel nützt}

Verbessert die Zugabe von Docetaxel das Überleben von Patientinnen mit Brustkrebs? Und sollte sie dann vor der klassischen Therapie erfolgen oder zeitgleich? 8-Jahres-Daten einer großen Studie geben Antworten.

$B_{n}^{c}$ ei Frauen mit nodal positivem Mammakarzinom begann die Breast International Group 1998 zu untersuchen, wie sich die Zugabe von Docetaxel zu einer Doxorubicin-basierten Chemotherapie auswirkt. Nach fünf Jahren zeigte sich ein Trend hin zu einem verbesserten krankheitsfreien Überleben (DFS) unter Docetaxel. Nun liegen 8-Jahres-Daten vor.

Die Patientinnen wurden in vier Gruppen randomisiert:

1. Sequenzielle Kontrollgruppe: Doxorubicin $\left(\mathrm{A} ; 75 \mathrm{mg} / \mathrm{m}^{2}\right) \times 4$, gefolgt von klassischem CMF (Cyclophosphamid, Methotrexat und 5-Fluorouracil)
2.Zeitgleiche Kontrollgruppe: AC (60 bzw. $\left.600 \mathrm{mg} / \mathrm{m}^{2}\right)$ x 4, gefolgt von CMF

3. Sequentielle Docetaxel-Gruppe: A (75 $\left.\mathrm{mg} / \mathrm{m}^{2}\right) \times 3$, gefolgt von Docetaxel $(\mathrm{T}$; $\left.100 \mathrm{mg} / \mathrm{m}^{2}\right) \times 3$, gefolgt von CMF

4. Gleichzeitige Docetaxel-Gruppe: AT (50 bzw. $75 \mathrm{mg} / \mathrm{m}^{2}$ ) x 4, danach CMF

Bei 2.878 Patientinnen hatte es nach median 93,4 Monaten 916 DFS-Ereignisse gegeben. Im primären Vergleich gab es keine signifikante Verbesserung im DFS durch Docetaxel (Hazard Ratio [HR] 0,91). Sekundäre Vergleiche aber zeigten, dass sequenzielles Docetaxel im Vergleich zur sequenziellen Kontrollgruppe das DFS signifikant verbesserte (HR 0,81 ). Darüber hinaus war das Gesamtüberleben unter sequenziellem Docetaxel besser als unter AT $(\mathrm{HR}=0,79)$.

Die besseren Ergebnisse der sequenziellen Gabe mögen auf die hier verwendete höhere Dosis von Docetaxel (100 statt $75 \mathrm{mg} / \mathrm{m}^{2}$ ) zurückzuführen sein. Eine Rolle könnten auch die höhere Do-
xorubicin-Dosis spielen (60 gegenüber $50 \mathrm{mg} / \mathrm{m}^{2}$ ) sowie die längere Behandlungsdauer (30 gegenüber 24 Wochen).

Die beste Prognose hatten Patientinnen mit Mammakarzinomen vom Typ Luminal-A. Für alle anderen Subtypen ergaben die Risikoquotienten einen günstigen Einfluss von sequenziell verabreichtem Docetaxel; allerdings gab es hier keine Signifikanz aufgrund der zu kleinen Probandenzahl.

Fazit: Im längeren Follow-up resultierte das sequenzielle Docetaxel-Regime in einem signifikant besseren Gesamtüberleben als zeitgleiches AT; sequenzielles Docetaxel zeigte auch weiterhin ein besseres DFS als die sequenzielle Doxorubicin-basierte Kontrolle. Christina Berndt

Oakman C et al. Overall survival benefit for sequential doxorubicin-docetaxel compared with concurrent doxorubicin and docetaxel in node-positive breast cancer - 8-year results of the Breast International Group 02-98 phase III trial. Ann Oncol. 2013 Jan 4. [Epub ahead of print]

\title{
Pluspunkte durch längere Tamoxifengabe bei ER-positivem Brustkrebs
}

\section{Seit mehr als einer Dekade ist die Tamoxifentherapie über zwei und fünf Jahre Standardoption bei Östrogenrezeptor-positivem Brustkrebs im Frühstadium. Unklar war, welchen Effekt eine Verlängerung auf zehn Jahre hat.}

m vergangenen Jahr stellte sich heraus, dass der Effekt einer 5-jährigen Tamoxifen-Therapie auf die Mortalität mindestens zehn Jahre anhält. Die ATLAS (Adjuvant Tamoxifen: Longer Against Shorter)-Studie zeigt nun, dass die Verdoppelung der Therapiedauer von fünf auf zehn Jahre Rezidiv- und Sterberaten weiter reduziert. Das kumulative Rezidivrisiko lag bei Therapieverlängerung in den Jahren 5-14 bei 21,4\%, in der Vergleichsgruppe ohne Verlängerung bei $25,1 \%$. Auch die Sterberate war mit $12,2 \%$ versus $15 \%$ niedriger.

In der Studie mit fast 13.000 Frauen mit Brustkrebs im Frühstadium hatten 6.846 Patientinnen Östrogenrezeptorpositive Karzinome. 3.428 von ihnen wurden zehn Jahre lang mit Tamoxifen behandelt, 3.418 Patientinnen beendeten die Therapie bereits nach fünf Jahren. Die Zahl der Rezidive betrug 617 mit und 711 ohne Therapieverlängerung (relatives Risiko 0,84; $\mathrm{p}=0,002$ ). Auch die krebsspezifische Mortalität war bei Therapieverlängerung niedriger (331 vs. 397 Todesfälle), ebenso die Gesamtmortalität (639 vs. 722 Todesfälle). Dabei wurde der Haupteffekt erst in der zweiten Dekade nach Diagnose beobachtet.

Auf der Basis der Ergebnisse von Metaanalysen, in denen Studien mit Tamoxifen über fünf Jahre berücksichtigt wurden sowie der ATLAS-Studie, haben die Wissenschaftler den Effekt abgeschätzt, den eine Tamoxifen-Behandlung über zehn Jahre im Vergleich zu keiner Tamoxifen-Therapie haben würde. Danach würde die krebsbedingte Mortalität nach zehn Jahren TamoxifenBehandlung, verglichen mit der Sterblichkeit ohne diese Therapie, in der zweiten Dekade nach der Diagnose fast halbiert werden.
Wichtigste unerwünschte Wirkung der verlängerten Tamoxifen-Therapie ist das Risiko eines Endometriumkarzinoms. In der ATLAS-Studie betrug das kumulative Risiko dafür in den Jahren 5-14 bei Frauen mit verlängerter Tamoxifen-Therapie 3,1\% im Vergleich zu nur 1,6\% in der Kontrollgruppe.

Fazit: Die noch vorläufigen Ergebnisse der ATLAS-Studie lassen vermuten, dass es durch eine verlängerte Tamoxifen-Therapie von fünf auf zehn Jahre gelingt, bei Frauen mit Östrogenrezeptorpositivem Brustkrebs im Frühstadium sowohl die Rezidivrate als auch die Mortalität weiter zu senken. Die Reduktion der brustkrebsspezifischen Sterberate wiegt das bereits niedrige Sterberisiko aufgrund eines Endometriumkarzinoms auf. Peter Leiner

Davies $C$ et al. Long-term effects of continuing adjuvant tamoxifen to 10 years versus stopping at 5 years after diagnosis of oestrogen receptorpositive breast cancer: ATLAS, a randomised trial. Lancet. 2012 Dec 4. [Epub ahead of print] 